\title{
Optical Interferometry Diagnostics in Laser-Driven Equation of State Experiments
}

\author{
D. M. Gold, P. M. Celliers, G. W. Collins, \\ L. B. DaSilva, R. C. Cauble, D. H. Kalantar, \\ S. V. Weber, and B. A. Remington \\ This paper was prepared for submittal to the \\ 11th Topical Conference on Shock Compression in Condensed Matter \\ Snowbird, Utah \\ June 27 - July 2, 1999
}

June 18, 1999

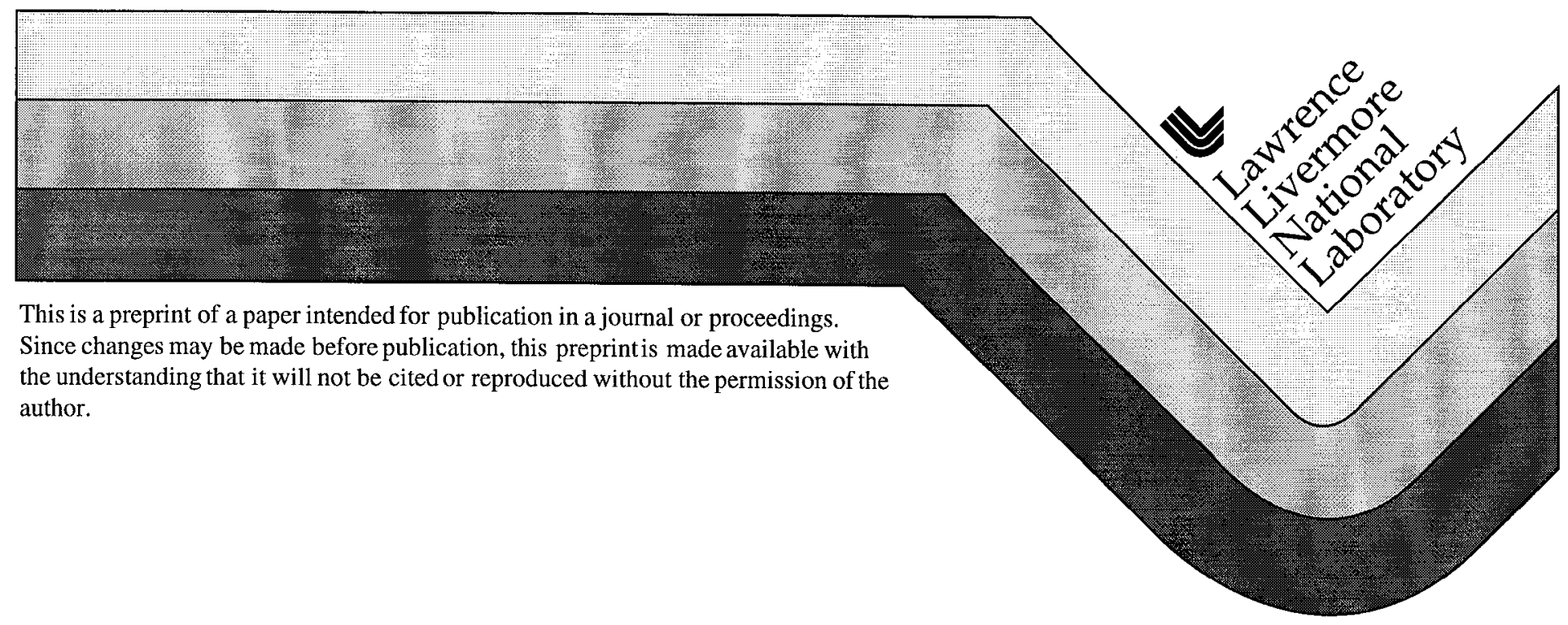


DISCLAIMER

This document was prepared as an account of work sponsored by an agency of the United States Government. Neither the United States Government nor the University of California nor any of their employees, makes any warranty, express or implied, or assumes any legal liability or responsibility for the accuracy, completeness, or usefulness of any information, apparatus, product, or process disclosed, or represents that its use would not infringe privately owned rights. Reference herein to any specific commercial product, process, or service by trade name, trademark, manufacturer, or otherwise, does not necessarily constitute or imply its endorsement, recommendation, or favoring by the United States Government or the University of California. The views and opinions of authors expressed herein do not necessarily state or reflect those of the United States Government or the University of California, and shall not be used for advertising or product endorsement purposes. 


\title{
OPTICAL INTERFEROMETRY DIAGNOSTICS IN LASER- DRIVEN EQUATION OF STATE EXPERIMENTS*
}

\author{
D.M. Gold, P.M. Celliers, G.W. Collins, L.B. DaSilva, R.C. Cauble, \\ D.H. Kalantar, S.V.Weber, B.A. Remington
}

Lawrence Livermore National Laboratory, Livermore CA 94550

\begin{abstract}
We have developed and tested several optical interferometric diagnostics to measure preheat and shock velocity in high-pressure equation of state experiments on the Nova laser. Theory and practical application of interferometric measurement techniques with illustrative experimental results are presented.
\end{abstract}

\section{INTRODUCTION}

Quantifying preheat and shock velocity are critical components in achieving accurate results with laserdriven shock compression experiments. Target preheat can significantly change a shocked material's density-temperature trajectory from the Principle Hugoniot. ${ }^{1}$ Analysis of off-Hugoniot shocks can be considerably more difficult and inaccurate. Preheat is a particular concern for lasergenerated shocks since the laser-matter interaction can produce penetrating $\mathrm{x}$-rays and suprathermal electrons. We have tested a variety of interferometric techniques using optical laser probing which give direct, high-precision measurements of target expansion due to preheat as well as shock timing, structure and velocity information. Expansion measurements, when combined with target models, quantify preheat levels and set upper limits on laser intensities for reliable data. We have also tested a VISAR ${ }^{2,3}$ velocity interferometer for observing the velocity of shocks in flight in transparent targets. ${ }^{4}$ This paper will focus on preheat measurements using two versions of displacement interferometry. The second technique is closely related to VISAR.

\section{EXPERIMENTAL DESIGN}

Two interferometric methods were used to quantify preheat in experiments at the Nova laser facility: Michelson ${ }^{5,6}$ interferometry and a novel double-passed velocity interferometer. Details of each setup are described below.

In all experiments the laser source was an injection-seeded, $>10 \mathrm{~ns} \mathrm{Ti}$ :Sapphire regenerative amplifier operating at wavelengths of $800 \mathrm{~nm}$ or $400 \mathrm{~nm}$ (frequency doubled). These wavelengths are anharmonic to the Nova laser allowing signal discrimination by optical filtering. The laser was propagated into the Nova target chamber and interferometer either via a relay image system (Michelson) or with a $1 \mathrm{~mm}$ diameter optical fiber and

\footnotetext{
${ }^{*}$ This work was performed by Lawrence Livermore National Laboratory under the auspices of the U.S. Department of Energy under Contract No. W-7405-ENG-48.
} 
relay image system (velocity interferometers). The target surface was relay imaged at $f / 3$ or $f / 10$ collection onto the slit of an optical streak camera (S-1 or S-20 photocathodes) with a magnification of 20 , resolution $<10 \mu \mathrm{m}$, and illumination area on the target of $1 \mathrm{~mm}$.

\section{DISPLACEMENT INTERFEROMETRY}

Preheat is quantified by measuring the displacement of the rear surface of a laserdriven target before shock breakout. Surface displacement produces a phase change in the laser probe pulse. This phase-shifted pulse, coherently interferes with a reference pulse changing the phase difference between them and shifting their interference fringe pattern. The displacement time history is recorded on a streak camera as a fringe pattern which shifts in time. Volumetric heating is then inferred from thermal expansion models.

\section{Michelson Interferometer}

A Michelson interferometer compares the target surface position to a fixed reference surface. The phase shift $\phi(t)$ of the target probe at position $x(t)$ is

$$
\phi(t)=\frac{4 \pi}{\lambda} x(t)
$$

One fringe shift occurs when the reflecting target surface has moved half a probe wavelength $(\lambda / 2=200 \mathrm{~nm})$. High quality fringes can be analyzed to an accuracy of 0.2 fringes or $40 \mathrm{~nm}$. This gives a sensitivity of a few hundred degrees Kelvin for most materials. An example of

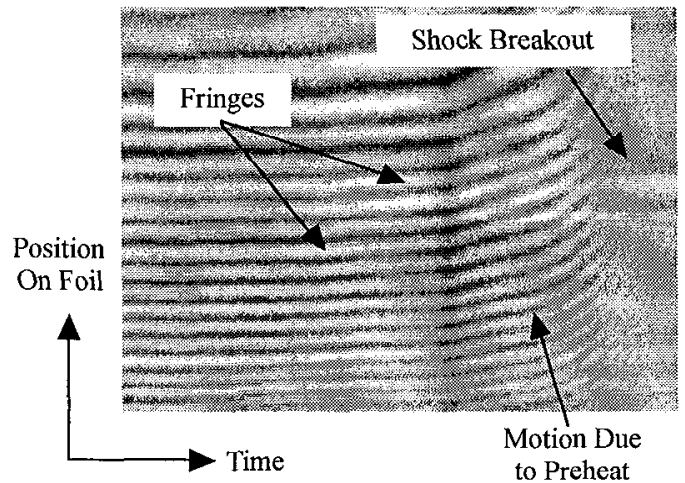

FIGURE 1. Streaked image of Michelson interferometer data.

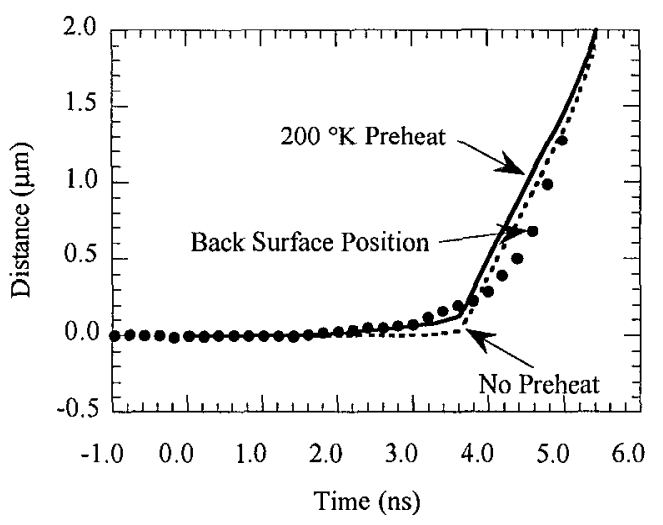

FIGURE 2. Reduced Michelson data with simulations.

preheat data in a $17 \mu \mathrm{m} \mathrm{Al}$ foil with hohlraum drive is shown in Fig. 1. Figure 2 shows the reduced data with simulations. Note that the fringes disappear upon shock breakout. On a stepped target we can obtain an average velocity (assuming a steady shock) from the stepped breakout times. Thus, preheat, spatial, shock structure and velocity are simultaneously revealed in these measurements. We extract the phase, $\phi$, from a fit of the observed fringe intensity $I$ to a time and space dependent sinusoidal function

$$
I(x, t)=A(x, t)+B(x, t) \sin (\phi(x, t))
$$




\section{Double-Passed VISAR Interferometer}

A double-pass VISAR ${ }^{7}$ geometry achieves displacement information by referencing a probe pulse reflected from a displaced surface position to a high fidelity copy of this pulse reflected at an earlier time before motion began. The interferometer is shown in Fig. 3. The laser pulse enters the input interferometer from the collimated output of a fiber and is split equally between a short arm and a long arm. The long arm is a $4 \mathrm{~m}$ air path delay consisting of a $1 \mathrm{~m}$ achromat and two $1 \mathrm{~m}$ radius of curvature spherical mirrors. This is substituted for the standard VISAR etalon because it provides a long (13.3ns) delay in a convenient, adjustable geometry. As in conventional VISAR, the image plane (virtual mirror) of the long arm and the short arm are equidistant from the beamsplitter.

The system is triggered so that the first pulse reaches the target a time $\tau$ before the Nova drive. The pulse sequence is illustrated in Fig. 4. The second pulse arrives during the drive and is phase shifted by reflecting off a target surface which has expanded from preheating. The two pulses then return to the output

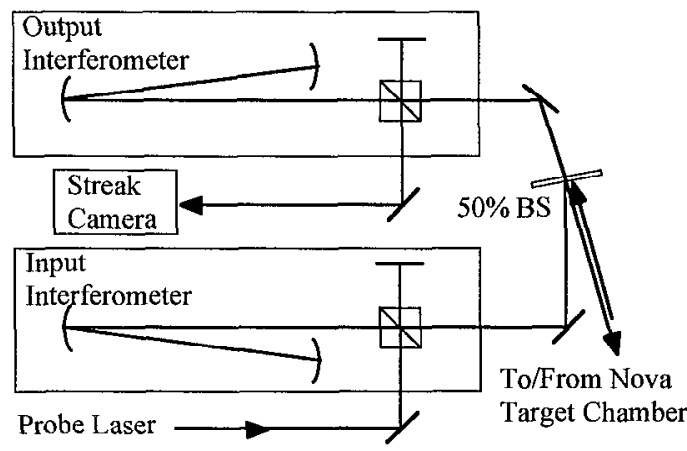

FIGURE 3. Double-pass VISAR interferometer setup.

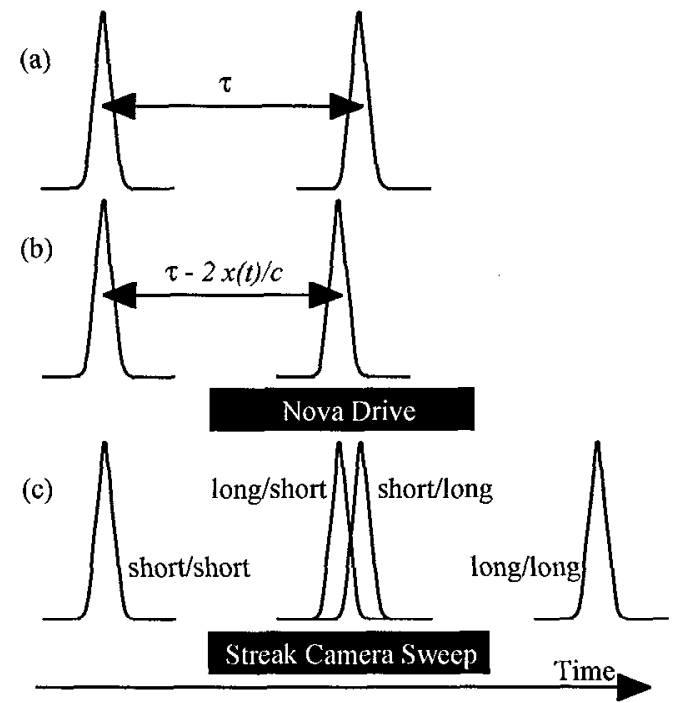

FIGURE 4. Time sequence for double-pass VISAR. (a) After input interferometer (b) During reflection from target (c) After output interferometer with the path of each pulse (input interferometer/output interferometer) indicated.

interferometer and are each split equally again with a copy being produced at exactly the same time delay, $\tau$, as before. Two of the four pulses produce the interferogram and the other two are not recorded because they lie outside the temporal window of the streak camera. The net optical phase difference between the interfering pulses is identical to the Michelson interferometer (Eq. 1). Note that the Michelson measures the target position relative to a fixed independent reference surface. Here the target acts as its own reference surface. To relate to the more conventional single-pass VISAR systems we assume a nearly constant expansion speed so $x(t)=v(t) t$ with $(\tau)(d v / d t)<v$ and the phase becomes

$$
\phi\left(t+\frac{1}{2} \tau\right)=\frac{4 \pi}{\lambda}\left\langle v\left(t+\frac{1}{2} \tau\right)\right\rangle \tau
$$


where the brackets denote a time average over $\tau$. This is the familiar VISAR velocity equation. ${ }^{2}$ When strong accelerations occur in a time $<<\tau$ the double-pass VISAR functionally measures displacements and the average velocity in Eq. 3 is not useful. Preheat experiments typically have acceleration periods of $\sim 1 \mathrm{~ns}$ which is much less than our interferometer delay of $\tau=13.3$ ns.

\section{DISCUSSION}

Some general pragmatic conclusions can be drawn as a result of these studies about the use of optical interferometry in laser-driven shock experiments. A VISAR-based configuration has many practical advantages. The entire interferometcr is outside the vacuum chamber giving greater stability and simpler alignment. VISAR will also work with non-specular (rough) reflecting surfaces so the driven samples do not need special preparation, polishing or precision mounting. Because VISAR interferometers work with spatially incoherent light, fiber delivery is possible. This is a great advantage in large facilities where the laser source may be far from the target and detector.

With either interferometer a high collection optical system is necessary. Precision alignment of the target surface to the optical system was often not possible bccause the Nova drive beams governed the target alignment. A fast objective alleviated this problem, but also raised signal to noise issues. When changing from $\mathrm{f} / 10$ (Michelson) to $\mathrm{f} / 3$ (VISAR) collection, streak camera retrace from late shock breakout plasma emission became of the order of the signal. The optimum solution to this tradeoff is a powerful probe pulse, high collection, and a gated streak camera to reject the later plasma emission.

Finally, fringe measurements give very precise values, however their accuracy is limited by the quality of heating models used for these systems. Heating in simple, one-component targets is easily estimated. Multilayer targets, however, require sophisticated modeling. Since the preheat detector only measures the displacement of the rear surface information on the expansion of each layer must be obtained by accurate modeling of the entire system. Nevertheless, we believe that the value of preheat measurement lies not in obtaining absolutely accurate temperatures, but in rejecting laser driven experiments with approximate preheat numbers which clearly invalidate a simple Principle Hugoniot analysis.

\section{REFERENCES}

${ }^{1}$ Zel'dovich, Ya. B., and Raizer, Yu. P., Physics of Shock Waves and High-Temperature Hydrodynamic Phenomena, Academic Press, New York, 1967.

${ }^{2}$ Barker, L.M., and Hollenbach, R.E., J. Appl. Phys. 43, 4669-4675 (1972).

${ }^{3}$ P. Celliers, G. W. Collins, L. B. Da Silva, D. M. Gold, and R. Cauble App. Phys. Lett. 73, 1320-1322 (1998).

${ }^{4}$ Collins, G.W., et. al., "Diamond Shocked toward a Metallic State," and DaSilva, L.B., et. al., "Equation of State Measurements of Lithium Fluoride at Mbar Pressures" to be published in Shock Compression in Condensed Matter-1999, edited by M.D. Furnish, AIP Conference Proceedings, New York 1999.

${ }^{5}$ Michelson, A.A., Amer. J. Sci. (3), 22, 120 (1881).

${ }^{6}$ Barker, L.M. and Hollenbach, R.E., Rev. Sci. Instrum. 36, 1617-1620 (1965).

${ }^{7}$ Erskine, D.J. and Holmes, N.C., Nature 377, 317-320

(1995). 